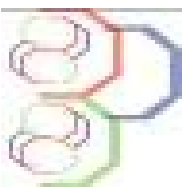

ISSN 1997-5902

\title{
Diversité et critères d'adoption des cultivars de maïs (Zea mays L.) dans le village Zounnou, Centre Bénin
}

\author{
Emile N. HOUNGBO \\ Agroéconomiste, Université d'Agriculture de Kétou, 05 BP 774 Cotonou (République du Bénin), \\ Tél.67763722 / 95246102, E-mail : enomh2@yahoo.fr
}

Original submitted in on $4^{\text {th }}$ November 2015. Published online at www.m.elewa.org on 31st December 2015 http://dx.doi.org/10.4314/jab.v96i1.6

\section{RÉSUMÉ}

Objectif : L'objectif de ce papier est de caractériser les cultivars de maïs et de relever les critères soutenant leur adoption.

Méthodologie et résultats : La démarche méthodologique a inclus une séance focus group et des enquêtes avec questionnaire de 80 producteurs de maïs, dont 32 femmes, dans le village Zounnou, centre Bénin. II se dégage que trois cultivars de maïs sont adoptés par les producteurs. II s'agit des cultivars Gbadé vôvô, gbadé wéwé et Carder. Gbadé Vôvô est adopté par $97,5 \%$ des producteurs, pendant que Gbadé wéwé et Carder sont adoptés respectivement par $75,0 \%$ et $37,5 \%$ des producteurs. Les critères d'adoption des cultivars de maïs sont par ordre de préférence décroissante l'importance du cultivar dans l'alimentation du ménage, la demande sur le marché et la disponibilité des semences. II s'agit de critères soutenus par la préoccupation de souveraineté alimentaire des ménages et qui amènent les producteurs à protéger une diversité de cultivars. Le critère de rendement élevé n'est pas le plus important à Zounnou.

Conclusion et application des résultats: La prise en compte des résultats de cette étude permettra de distinguer les cultivars de maïs à fort potentiel d'adoption dans le village et les communautés similaires en vue de l'adaptation aux changements climatiques par le développement d'une agriculture résiliente, fondée sur l'agrobiodiversité et les écosystèmes.

Mots clés : Agrobiodiversité, maïs, cultivar, adoption, Bénin.

Diversity and Adoption Criteria of Cultivars of Maize (Zea mays L.) in Zounnou Village, Middle Benin

\begin{abstract}
Objective: The aim of this paper is to characterize the maize cultivars and identify the criteria supporting their adoption.

Methodology and results: The methodological approach included a focus group discussion and surveys with questionnaire of 80 farmers, including 32 women, in the Zounnou village, middle Benin. It revealed that three maize cultivars were adopted by farmers: Gbade vovo (red maize), Gbade wewe (white maize) and Carder. Gbade vovo was adopted by $97.5 \%$ of the farmers, while Gbade wewe and Carder were adopted respectively by $75.0 \%$ and $37.5 \%$ of the farmers. The adoption criteria of maize cultivars were in a decreasing order of preference the importance of the cultivar for household food, the demand on the market and the availability of good quality of seed. These criteria, which are supported by the
\end{abstract}


preoccupation of households' food sovereignty, lead farmers to protect a diversity of cultivars. The criterion of high yield was not the most important.

Conclusion and application of results: Taking into account the results of this study will help distinguishing the maize cultivars with high potential for adoption in the village and similar communities in order to adapt to climate change through the development of a resilient agriculture based on agro-biodiversity and ecosystems.

Keywords: Agro-biodiversity, Maize Cultivar, Adoption, Benin.

\section{INTRODUCTION}

L'adaptation aux changements climatiques impose la nécessité de développer une agriculture résiliente, fondée sur l'agrobiodiversité et les écosystèmes. En effet, l'agrobiodiversité confère à l'ensemble du système de production une résistance, une tolérance et une résilience face aux facteurs biotiques et abiotiques de l'environnement de culture. Elle contribue à assurer une alimentation diversifiée et d'autres fonctions socioéconomiques (Baco, 2007). La Convention sur la Diversité Biologique (Nations Unies, 1992) indique dans son préambule qu'un grand nombre de communautés locales et de populations autochtones dépendent étroitement et traditionnellement des ressources biologiques sur lesquelles sont fondées leurs traditions et qu'il est souhaitable d'assurer le partage équitable des avantages découlant de l'utilisation des connaissances, innovations et pratiques traditionnelles intéressant la conservation de la diversité biologique et l'utilisation durable de ses éléments. La diversité du maïs, céréale la plus consommée au Bénin, loin devant le riz et le sorgho (ONS, 2010), est donc capitale pour garantir la sécurité alimentaire durable des populations. En effet, d'après Adégbola et al. (2011), le maïs est utilisé au Bénin sous plusieurs formes suivant les différentes destinations. En alimentation humaine, les modes de consommations diffèrent suivant les régions et les catégories sociales (produit frais ou vert, graines sèches décortiquées et cuites, graines sèches moulues en farine ou semoule). Dans l'alimentation du bétail, le maïs entre dans la composition des provendes et des sous produits tels que les sons, les tourteaux et germes. Les tiges et feuilles ensilées sont également utilisées pour le bétail. Dans l'agro-industrie, le maïs entre dans la fabrication de boisson essentiellement la bière, les farines améliorées infantiles et adultes. Habituellement cultivé au sud et au centre du Bénin, la production de maïs s'est étendue aux zones de production de coton dans les régions septentrionales. Le volume de la production a franchi la barre des 800.000 tonnes en 2004 et celle de 1 million de tonnes en 2009. Le maïs, première production céréalière dans le monde (N'Da et al., 2013 ; Brassaler, 2010), fait d'ailleurs partie des treize (13) filières retenues dans le Plan stratégique de relance du secteur agricole $d u$ Bénin (PSRSA) par le Ministère de l'agriculture, de l'élevage et de la pêche (MAEP) à promouvoir pour assurer la sécurité alimentaire et la croissance économique du Bénin (MAEP, 2011). Mais, en dépit des conditions favorables dont jouit cette culture, force est de constater que la production de maïs connaît une évolution en dents de scie qui fait fluctuer son solde vivrier dans des proportions parfois inquiétantes. Ce qui fait peser des menaces tant sur la sécurité alimentaire que sur les revenus des producteurs. Les besoins d'utilisation spécifique du maïs (brasserie, sweet corn/légume, fourrage, etc.) ne sont pas encore couverts par la production locale. D'où, la nécessité d'engager des actions spécifiques en faveur de la filière maïs afin d'améliorer sa contribution à la sécurité alimentaire et aux revenus des producteurs (MAEP, 2011). L'une de ces actions reste la préservation de la diversité des cultivars ; ce qui ne peut se faire sans la prise en compte des savoirs endogènes en la matière. Car, la diversité biologique renforce la capacité d'adaptation des écosystèmes agricoles, en leur permettant de faire face à des perturbations de 
toutes sortes (érosion, aléas climatiques, pauvreté des sols, etc.) et de se maintenir en équilibre. L'objectif de ce papier est de caractériser les cultivars et relever les critères soutenant leur adoption. L'hypothèse de base est que les

\section{MATÉRIEL ET MÉTHODES}

Zone d'étude : Le village Zounnou est situé dans la Commune de Zagnanado au centre Bénin. La Commune de Zagnanado est classée dans la zone agroécologique $\mathrm{VI}$ (zone des terres de barre), caractérisée par la production de maïs, de manioc, d'arachide et de niébé comme principales spéculations; avec le maïs en tête de rotation. Dans cette zone, le régime des pluies est souvent perturbé, entrainant des changements dans les cycles de production annuels. D'une superficie de $55 \mathrm{~km}^{2}$, le village Zounnou compte une population d'environ 850 habitants, dont $52 \%$ de femmes, et composé en grande majorité d'ethnie Mahi. Le relief est accidenté, avec des versants à pentes plus ou moins fortes pat endroit et de petites collines. On y rencontre des sols ferralitiques et des sols hydromorphes. Ceux-ci occupent environ $60 \%$ de la superficie du village. L'agriculture est la principale activité à Zounnou. Les spéculations produites sont les cultures vivrières (riz, maïs, manioc, niébé, taro) et les cultures de rente (palmier à huile). Le maïs est l'aliment de base de la population et occupe $90 \%$ de leurs rations alimentaires (Akondé et al., 2013)

\section{RÉSULTATS}

Diversité des cultivars de maïs : Trois cultivars de maïs sont identifiés dans le village Zounnou. Ils se distinguent dans leurs présentations et dans leurs appellations par les populations. II s'agit des cultivars "Gbadé vôvô » (maïs rouge), " Gbadé wéwé » (maïs blanc) et « Carder». En dehors du cultivar Carder qui est une variété de maïs améliorée, les deux autres variétés (Gbadé wéwé et Gbadé vôvô) sont des variétés locales traditionnellement cultivées dans le village depuis des générations. Plus spécifiquement, les caractéristiques distinctives des trois cultivars sont les suivantes.

Le cultivar Gbadé vôvô: "Gbadé vôvô » est un cultivar que l'on reconnaît à travers ses épis de petite taille, portant de petits grains jaunes. Au plan agronomique, Gbadé vôvô est une variété à cycle de production court par rapport aux autres variétés. Sa récolte commence 2,5 mois après le semis. De ce fait, producteurs adoptent les cultivars sur la base d'une diversité de critères locaux favorables à l'agrobiodiversité pour la sécurité alimentaire et le bien-être des populations.

Collecte et analyse des informations : Les données collectées et informations ont été collectées de novembre à décembre 2013. La méthodologie utilisée a été d'abord participative. Cette option a été faite pour permettre l'expression libre des producteurs et une validation collective des diverses déclarations et appréciations. Elle permet aussi d'améliorer la fiabilité des déclarations au moyen de la triangulation des informations. La méthode a consisté à la conduite d'une séance focus group, complétée par des observations participantes et des entretiens individuels en vue de la vérification de certaines déclarations. L'analyse qualitative a été complétée par une enquête avec questionnaire de 80 producteurs de maïs, dont 32 femmes, pour recueillir des informations spécifiques. Les données qualitatives ont été analysées au fur et à mesure de leur collecte. Les données d'enquête ont fait l'objet de dépouillement, puis de calcul de paramètres statistiques simples comme la moyenne, la proportion et l'erreur-standard; l'option de la recherche étant essentiellement descriptive.

elle est plus adaptée à la petite saison des pluies (soit de mi-septembre à mi-novembre) parce qu'elle n'exige pas beaucoup d'eau. II faut souligner que la petite saison des pluies vient peu après la période de soudure pendant le bilan vivrier est souvent critique. Le cultivar Gbadé vôvô est aussi bien résistant aux attaques des ravageurs post-récolte (les charançons notamment). C'est un cultivar dont le stockage et la conservation après récolte ne posent pas de grands problèmes selon les producteurs. L'épi du cultivar Gbadé vôvo est présenté dans la photo 1. 


\section{Houngbo N.Emile. J. Appl. Biosci. 2015 Diversité et critères d'adoption des cultivars de maïs (Zea mays L.) dans le village Zounnou, Centre Bénin}

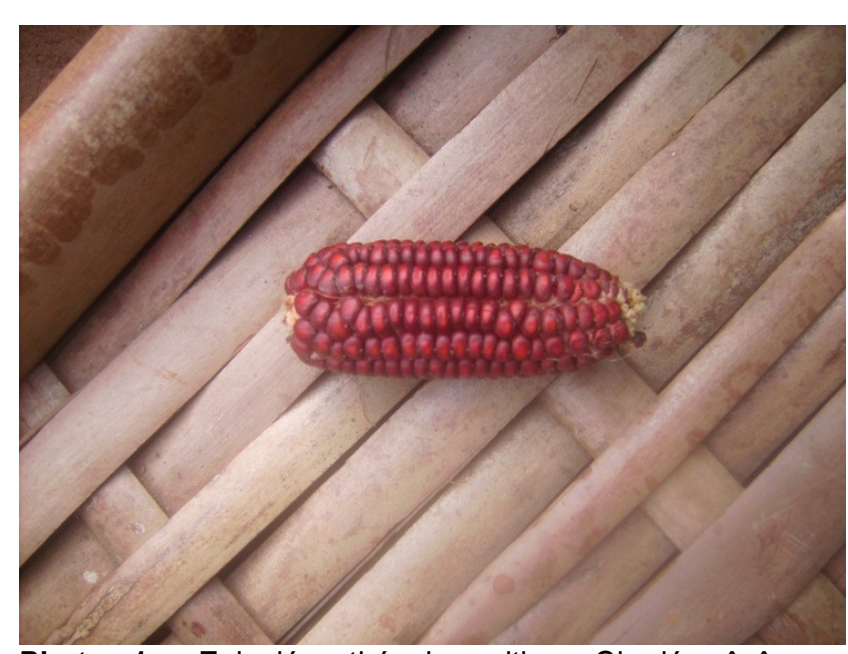

Photo 1: Epi déspathé du cultivar Gbadé vôvô (novembre 2013)

Le cultivar " Gbadé wéwé » : Le cultivar Gbadé wéwé présente de petits épis ou des épis de moyenne taille, portant de petits grains blancs. Gbadé wéwé a un cycle de production plus long que celui de Gbadé vôvô. Sa récolte commence 3,5 mois après le semis. De ce fait, c'est une variété de maïs adaptée à la grande saison des pluies, soit de mi-mars à mi-juillet. Suivant les propos de certains enquêtés (80\%), lorsqu'elle est cultivée en petite saison des pluies, elle ne donne pas un rendement intéressant. II faut souligner que sur l'échantillon étudié, $50 \%$ des enquêtés ne cultivant pas cette variété signalent les difficultés de stockage et de conservation du cultivar Gbadé wéwé après les récoltes. C'est une variété de maïs très attaquée par les charançons. Cette réalité justifie le fait que $25 \%$ des producteurs de maïs dans le village, ne donnent pas une bonne appréciation de ce cultivar. La photo 2 présente des épis du cultivar Gbadé wéwé.
Photo 2 : Deux épis déspathés du cultivar Gbadé wéwé (novembre 2013)

Le cultivar "Carder »: Le cultivar Carder, contrairement aux variétés locales, présentent de gros épis à gros grains de couleur uniforme jaune ou blanc. ॥ est identifié en réalité au regard de l'institution publique d'encadrement des producteurs qui le vulgarise: le Centre d'action régional pour le développement rural (CARDER). C'est donc un cultivar de variété améliorée issu de la recherche agronomique et jugé a priori avantageux pour le producteur. Les variétés Carder ont un cycle de production plus long comparé à la variété locale Gbadé vôvô. Leur récolte commence 3 à 4 mois après le semis. Ce sont des variétés exigeantes en intrants (engrais notamment) et en eau. Pour certaines de ces variétés, les tiges, même à une petite taille, donnent des épis de maïs; ce qui favorise les rongeurs qui ne rencontrent quasiment pas de difficultés pour ravager les cultures. Cette caractéristique explique le fait que la culture des variétés Carder a été substituée progressivement par la culture du riz, plus rentable que le maïs selon les propos recueillis lors de la séance focus group. Les photos 3 présentent des épis du cultivar Carder. 


\section{Houngbo N.Emile. J. Appl. Biosci. 2015 Diversité et critères d'adoption des cultivars de maïs (Zea mays L.) dans le village Zounnou, Centre Bénin}
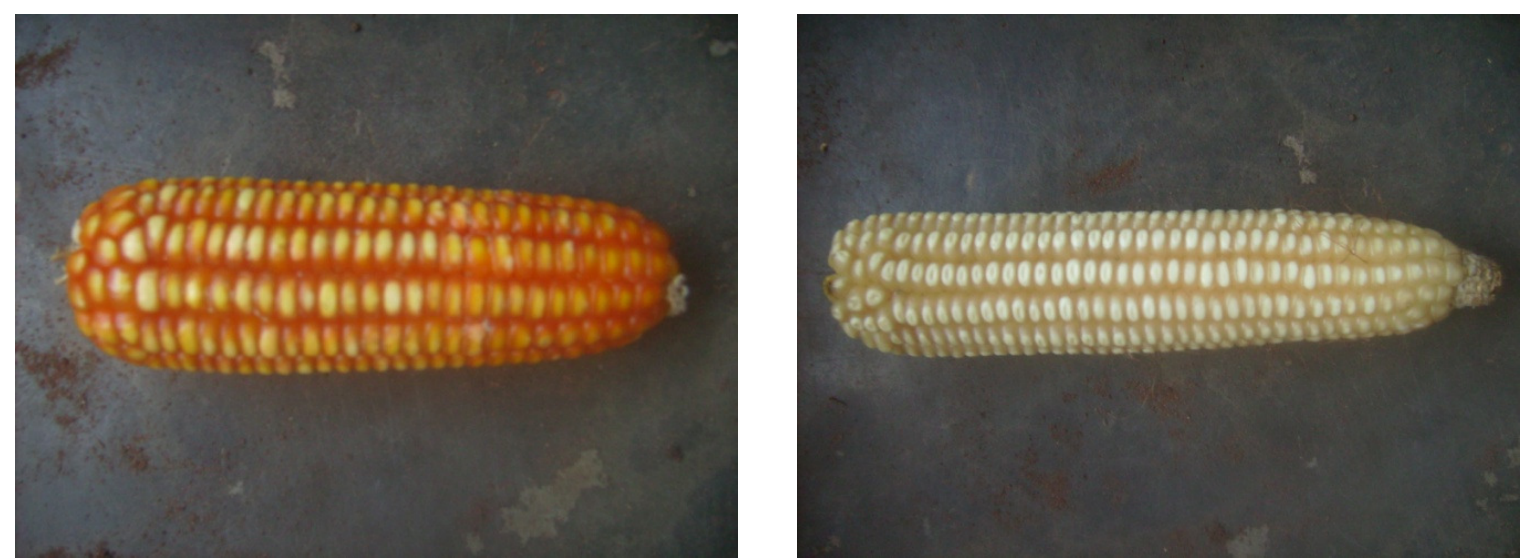

Photo 3 : Deux épis déspathés de couleur jaune (à gauche) et blanc (à droite) (novembre 2013)

Déterminants de l'adoption des cultivars : On relève trois critères importants qui motivent les producteurs à la préservation de l'agrobiodiversité du maïs dans le village Zounnou. Les producteurs privilégient par ordre d'importance décroissante l'importance du cultivar dans l'alimentation des ménages, la demande sur le marché et la disponibilité des semences. Ainsi, le cultivar Gbadé vôvô plus destinée à l'autoconsommation qu'à la vente à cause de la couleur de ses grains est d'une grande utilité dans le village. Gbadé vôvô s'avère le cultivar de maïs dominant dans le village. En effet, il est le plus apprécié et le plus succulent selon les déclarations des producteurs. La consommation de Gbadé vôvô permettrait de résoudre les problèmes de malnutrition chez les enfants. Cependant, c'est un cultivar qui est moins demandé sur les marchés puisque sa pâte et sa bouillie sont d'une couleur semblable au jaune d'œuf, donc non blanche. Cette caractéristique constitue d'un côté, la raison poussant certains producteurs à délaisser sa culture. Tous les enquêtés ne cultivant pas Gbadé vôvô affirment que la plupart des produits dérivés issus de sa transformation sont moins demandés par les consommateurs sur le marché, à l'exception de ses épis frais grillés et cuits qui sont en revanche bien vendus sur le marché. Comparé à Gbadé vôvô, le cultivar Gbadé wéwé est plus demandé sur le marché, car sa bouillie, son akassa et sa pâte sont de couleur blanche, très appréciés par les consommateurs. Ainsi, sa production est plus destinée à la commercialisation qu'à l'autoconsommation. Selon les enquêtés, le cultivar Carder donne un rendement meilleur à ceux de Gbadé vôvô et de Gbadé wéwé, mais il se trouve handicapé par les difficultés que pose l'accès à ses semences de bonne qualité. II n'a pas encore bien intégré la banque de semences des producteurs. Car, la taille et le poids des épis, de même que la grosseur des grains sont à l'avantage de ce cultivar. Plus que le rendement élevé, l'accès facile aux semences de bonne qualité représente donc un préalable indispensable à l'adoption massive des cultivars par les producteurs. Ceci suggère qu'au-delà de la sécurité alimentaire, les producteurs accordent du prix à la souveraineté alimentaire.

Niveaux d'adoption des cultivars : Comme énoncé plus haut, le cultivar Gbadé vôvo est le cultivar le plus cultivé dans le village Zounnou. II est largement adopté devant le Carder qui pourtant donne le meilleur rendement. La figure 1 présente la fréquence d'adoption des trois cultivars de maïs dans le village. 


\section{Houngbo N.Emile. J. Appl. Biosci. 2015 Diversité et critères d'adoption des cultivars de maïs (Zea mays L.) dans le village Zounnou, Centre Bénin}



Figure 1 : Fréquence d'adoption des cultivars de maïs à Zounnou

II ressort donc que $97,5 \%$ des enquêtés cultivent Gbadé vôvô, contre seulement $37,5 \%$ pour le cultivar Carder. Ainsi Gbadé vôvô et Gbadé wéwé sont dans cet ordre les deux premières variétés les plus cultivées dans le village. Le cultivar Gbadé vôvô est cultivé sur une superficie moyenne de $1,36 \pm 0,161$ ha par saison dans le village. Les superficies affectées aux cultivars

\section{DISCUSSION}

Critères de sauvegarde paysanne de la biodiversité et pauvreté : Les producteurs retiennent des cultivars suivant des critères diversifiés. On note par ordre d'importance décroissante l'importance du cultivar dans l'alimentation du ménage, la demande sur le marché et la disponibilité des semences. Ces résultats bien que proches, sont différents de ceux obtenus par Baco et al. (2010) sur les variétés de maïs au nord Bénin. Ces auteurs avaient identifié 4 à 6 cultivars dans chacun des six villages étudiés; ce qui suggère que le nombre de cultivars de maïs au Bénin doit être élevé. Ils trouvaient que les variétés de maïs étaient adoptées à $64,67 \%$ pour le rendement, à $12 \%$ pour la précocité et à $9,33 \%$ pour la résistance à la sécheresse. La résistance à l'inondation par exemple était l'un des critères les moins importants. Ces différences de critères observées entre le centre et le nord Bénin suggèrent que les critères d'adoption de cultivars doivent être placés dans leur contexte agronomique et socioéconomique local. Les réalités agronomiques et socioéconomiques déterminent ainsi les réactions d'adaptation des producteurs. Les observations de
Gbadé wéwé et Carder sont respectivement de 0,944 \pm 0,224 ha et de $0,5 \pm 0,127$ ha. II faut rappeler que le village Zounnou se trouve dans la zone de forte pression foncière du bas Bénin où la superficie moyenne exploitée est largement en deçà de 5 ha, une moyenne attribuée à la région nord du pays.

Baco et al. (2010) sont quasiment celles du Comité éditorial d'AGRIDAPE (2014) qui affirme que devant l'accroissement de la population mondiale et par conséquent des besoins alimentaires, la productivité est devenue un critère de taille dans le choix des espèces exploitées dans l'agriculture. C'est souvent au nom d'un souci d'amélioration des rendements que les espèces améliorées, jugées "plus productives », sont introduites. Mais, leur diffusion à grande échelle a des répercussions néfastes sur l'avenir des espèces locales qui se retrouvent, du coup, négligées. Cette position est bien discutable, car elle semble se focaliser sur l'agriculture commerciale moins préoccupée par la sauvegarde de l'agrobiodiversité, contrairement à l'agriculture familiale. Or, c'est cette dernière forme de production agricole qui domine le monde et les populations traditionnelles sont les variétés les plus cultivées au Bénin (Abadassi, 2001). En effet, l'agriculture familiale se révèle être la forme incontournable d'agriculture qui domine le monde. Près de $98 \%$ des 500 millions de producteurs agricoles du monde sont des agriculteurs familiaux (CTA, 2014). 


\section{Houngbo N.Emile. J. Appl. Biosci. 2015 Diversité et critères d'adoption des cultivars de maïs (Zea mays L.) dans le village Zounnou, Centre Bénin}

L'agriculture familiale couvre $85 \%$ des terres agricoles en Asie, $83 \%$ en Amérique du Nord et du Centre, $68 \%$ en Europe, $62 \%$ en Afrique et $18 \%$ en Amérique du Sud. Elle occupe 1,3 milliard d'individus dans le monde, $40 \%$ des actifs du monde et $70 \%$ de la production alimentaire mondiale. Elle assure $98 \%$ de la production vivrière en Afrique et $100 \%$ de la production du coton et du cacao. Sur les 570 millions d'exploitations agricoles au monde, 500 millions sont des exploitations familiales, soit $87,7 \%$. L'agriculture familiale est à la base de la sauvegarde de la biodiversité agricole. Malheureusement, l'agriculture familiale est aussi le siège la pauvreté rurale dans le monde. On se demande comment valoriser au niveau des agriculteurs familiaux ces bonnes pratiques de sauvegarde de la biodiversité qui s'avèrent utiles pour faire face efficacement au phénomène de changement climatique actuel et réduire dans le même temps la pauvreté à leur niveau. En effet, les sources de la plus grande diversité de variétés tendent à être les zones pauvres, isolées et, souvent, peu productives du monde en développement, et que les agriculteurs qui conservent d'ordinaire le matériel génétique végétal traditionnel le plus diversifié sont susceptibles d'être les plus pauvres (Altieri, s.d.). L'agriculteur familial est encore, souvent, associé à pauvreté. Et pour cause, $70 \%$ des plus démunis au monde sont des agriculteurs familiaux ou pastoraux et $95 \%$ de ces exploitations familiales font encore aujourd'hui moins de 5 hectares (CTA, 2014). Mais, en basant une stratégie de développement rural sur l'agriculture traditionnelle et les connaissances ethnobotaniques, non seulement assure-t-on la poursuite de l'exploitation et la préservation de ressources génétiques précieuses, mais on permet de plus la diversification des stratégies de subsistance des paysans, y compris l'établissement de liens avec les marchés externes (Alcorn, 1984; Caballero \& Mapes, 1985). La promotion des agriculteurs familiaux à la fois

\section{CONCLUSION}

Trois cultivars de maïs sont adoptés dans le village Zounnou. Deux variétés traditionnelles (Gbadé vôvô et Gbadé wéwé) et un cultivar de variété améliorée mis au point par la recherche et introduit dans le village par l'institution nationale d'encadrement des producteurs qu'est le CARDER. Les critères d'adoption des cultivars de maïs sont divers et variés dans le village. Ces critères sont par ordre d'importance décroissante l'importance du cultivar dans l'alimentation du ménage, la demande sur le marché et la disponibilité des dans leurs pratiques agricoles vertueuses et dans la réduction substantielle de la pauvreté en leur sein demeure donc un défi.

Désignations et pratiques de maintien de la diversité des cultivars : Les paysans de Tagouana au Centre-Nord de la Côte d'Ivoire identifient les cultivars exclusivement sur la base de la coloration des grains. Deux noms vernaculaires ont été inventoriés pour désigner les trois variétés locales de maïs violet observées dans les différentes localités enquêtées. II s'agit des maïs à grains violet foncé, des maïs à grains violet clair et des maïs à grains violet clair qui tire sur le rouge (N'Da et al., 2013). Les appellations adoptées dans le village Zounnou sont semblables à celles que rapportent ces auteurs sur la Côte d'Ivoire, ne serait-ce que les variétés traditionnelles du village : Gbadé wéwé (maïs blanc) et Gbadé vôvô (maïs rouge). Une chose est d'adopter les cultivars, mais une autre est de s'enquérir des stratégies développées par les producteurs pour préserver l'agrobiodiversité. A ce sujet Kouakou et al. (2010) soulignent que les pratiques les plus répandues pour maintenir la biodiversité de ces variétés sont la culture polyvariétale et l'héritage des semences. L'une des pratiques les plus répandues de maintien de la biodiversité de maïs révélé par leur étude en Côte d'Ivoire est l'héritage des semences. Le Violet de Katiola est la variété de maïs la plus cultivée en raison de son caractère socioculturel important, sa bonne qualité organoleptique, sa valeur marchande et de ses vertus thérapeutiques. Le savoir faire des paysans de Katiola dans la gestion de la biodiversité et en particulier de la variété traditionnelle de maïs " Violet de Katiola ", a permis à ce peuple de maintenir de génération en génération cette variété prisée pour ses propres besoins. Tout porte donc à croire que les producteurs (familiaux) sont plus préoccupés par la souveraineté alimentaire de génération en génération.

semences. II s'agit de critères soutenus par la préoccupation de souveraineté alimentaire des ménages et qui amènent les producteurs à protéger une diversité de cultivars. Le critère de rendement élevé n'est pas le plus important à Zounnou, car le cultivar Carder à rendement plus élevé est le moins adopté. II pourrait le devenir si la condition d'accès aisé aux semences de bonne qualité était satisfaite. La situation observée au nord Bénin par d'autres auteurs n'a pas été la même. Ce qui suggère que la gestion de 


\section{Houngbo N.Emile. J. Appl. Biosci. 2015 Diversité et critères d'adoption des cultivars de maïs (Zea mays L.) dans le village Zounnou, Centre Bénin}

l'agrobiodiversité doit donc être abordée localement, suivant les réalités agronomiques et socioéconomiques

\section{RÉFÉRENCES BIBLIOGRAPHIQUES}

Abadassi J, 2001. Caractérisation de quelques variétés améliorées de maïs cultivées au Bénin, Bulletin de Recherche Agronomique 34, 1-6. http://www.slire.net/download/1198/abadassi_ bra 034 2001.pdf (consulté le 20/10/2015).

Adegbola PY, Aloukoutou A, Diallo B, 2011. " Analyse de la compétitivité du maïs local au Bénin ", Programme de Renforcement et de Recherche sur la Sécurité alimentaire en Afrique de l'Ouest (PRESAO), Composante SRAI, Résumé $\quad N^{\circ}$ 1- 2011-12 - Maïs, http://www.aec.msu.edu/fs2/presao.htm, (site consulté le 05/10/2015).

Akondé AF, Fantodji B, Sangninon C, Sodji M, Sourou VAB, 2013. Rapport de stage: Monographie en milieu rural, village Zounnou, Kétou: ENSTA-Kétou, 62 pp.

Alcorn JB, 1984. Huastec Mayan ethnobotany. Austin : University of Texas Press.

Altieri MA, (s.d.). Les aspects socioculturels de la diversité du maïs indigène, Université de la Californie à Berkeley, Secrétariat de la Commission de coopération environnementale de l'Amérique du Nord, $10 \mathrm{pp}$. http://www.cec.org/Storage/48/4004 Altierif.pdf (site consulté le 16/10/2015)

Baco MN, 2007. Gestion locale de la diversité cultivée au Nord Bénin : éléments pour une politique publique de conservation de l'agrobiodiversité de l'igname (Dioscorea spp), Thèse de doctorat, université d'Orléans, France, 406 pp.

Baco MN, Abdoulaye T, Sanogo D, Langyintuo A, 2010. Caractérisation des ménages producteurs de maïs en zone de savane sèche au Bénin, Rapport pays- Enquêteménage - Benin. IITA Ibadan, Nigeria, 38 p. en présence.

Bassaler N, 2000. Le maïs et ses avenirs, Cahier $N^{\circ} 13$ publié par le GERPA avec le concours d'Electricité de France, Mission Prospective ,47 pp.

Caballero JN. and Mapes CS, 1985. « Gathering and subsistence patterns among the P'urhepecha indians of Mexico ». J. Ethnobiol. 5 : 31-47.

CTA-Centre Technique de coopération Agricole et rurale, 2014. « Agriculture familiale. Le début d'une renaissance », Spore numéro horssérie, août 2014.

MAEP-Ministère de l'agriculture, de l'élevage et de la pêche, 2011. Plan stratégique de relance du secteur agricole (PSRSA), Cotonou: MAEP, 115 pp.

Kouakou CK, Akanvou L, Konan YA, Mahyao A, 2010. Stratégies paysannes de maintien et de gestion de la biodiversité du maïs (Zea mays L.) dans le département de Katiola, Côte d'Ivoire, Journal of Applied Biosciences 33: $2100-2109$.

Nations Unies, 1992. Convention sur la Diversité Biologique, 30 $\mathrm{pp}$, https://www.cbd.int/doc/legal/cbd-fr.pdf (consulté le 20/10/2015).

N'Da HA, Akanvou L, Kouakou CK, 2013. Gestion locale de la diversité variétale du maïs (Zea mays L.) violet par les Tagouana au CentreNord de la Côte d'lvoire, Int. J. Biol. Chem. Sci. 7(5): 2058-2068.

ONS-Office National de Soutien des revenus agricoles, 2010. Rapport d'étude du Prix plancher du maïs au titre de la campagne 2010-2011, Rapport d'étude, Cotonou: Ministère de l'Agriculture, de l'Élevage et de la Pêche (MAEP) ,79 pp. 